\title{
Compact boundary-condition-determined wave function for positronium hydride (PsH)
}

\author{
Dario Bressaninia ${ }^{a}$ and Gabriele Morosi ${ }^{b)}$ \\ Dipartimento di Scienze Chimiche, Fisiche e Matematiche, Universita' dell'Insubria, Sede di Como, \\ via Lucini 3, 22100 Como, Italy
}

(Received 26 June 2003; accepted 15 July 2003)

\begin{abstract}
A simple, compact, and accurate wave function for positronium hydride is written as a product of Pade' approximants for electron-nucleus interactions and of Jastrow functions for electron-electron interactions. Most of the parameters are fixed taking into account both the correct cusp conditions when two particles collide and the correct asymptotic behavior when one or two particles go to infinity. The remaining parameters were optimized by variational Monte Carlo calculations. The energy of this single term wave function is $-0.786073(6)$ hartree and favorably compares with very long configuration interaction expansions and even with explicitly correlated function expansions. The exam of the wave function and of various two-dimensional distribution functions shows that the PsH structure is similar to the hydrogen anion structure, with the positron slightly perturbing it and its motion strongly correlated to the electrons that are squeezed towards each other and towards the nucleus. (C) 2003 American Institute of Physics. [DOI: 10.1063/1.1605931]
\end{abstract}

\section{INTRODUCTION}

During the last few years, attention has been paid to the energetic and structural properties of systems containing one or more positrons, ${ }^{1}$ with the aim to elucidate the problem of their stability and the annihilation behavior of positrons in ordinary matter. ${ }^{2,3}$ There is a growing number of experimental techniques that can accurately probe the interaction between matter and antimatter, and the experiments need theoretical support to be interpreted. ${ }^{4}$ Positron containing systems represent a challenge for the standard methods of quantum chemistry [self-consistent field (SCF), configuration interaction (CI), density functional theory (DFT)] since they do not introduce electron-positron distances explicitly, and so they are unable to correctly reproduce the local behavior of the wave function when two particles collide. Quantum Monte Carlo methods can treat the instantaneous correlation between particles exactly and on equal footings, so they represent the ideal technique to study systems containing positrons. ${ }^{5,6}$

Here we focus our attention on the simplest system containing nuclei, electrons, and one positron and possessing a bound state: namely, PsH. Despite its molecularlike formula, $\mathrm{PsH}$ is an exotic atom. PsH is a very useful testing ground to study correlation effects between electrons and positrons, since the Hartree-Fock theory is not able to predict a bound state stable against the dissociation into Ps and $\mathrm{H}$. The SCF energy $^{7}$ is -0.6669 hartree, well above the dissociation limit of -0.75 hartree, and the SCF annihilation rate is eight times smaller than the exact one.

PsH stability was predicted by Ore, ${ }^{8}$ who used a simple correlated wave function and obtained a total energy of

\footnotetext{
a)Electronic mail: Dario.Bressanini@uninsubria.it

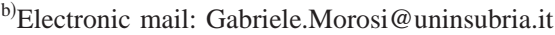

-0.75251 hartree, that is, a binding energy of 0.00251 hartree.

Later PsH was experimentally observed by Schrader et al. ${ }^{9}$ in collisions between positrons and methane, $e^{+}$ $+\mathrm{CH}_{4} \rightarrow \mathrm{CH}_{3}^{+}+\mathrm{PsH}$.

Starting from Ore's pioneering work, calculations of the total and binding energies have improved over the years. In general, correlation effects are so great that any method based on the independent particle model is completely inadequate in yielding accurate energies, structural properties, and annihilation rates. The configuration interaction method, although exact in the limit of a complete basis set, converges even more slowly than in electronic systems. The best CI results were obtained by Bromley and Mitroy, ${ }^{10}$ including 95324 configurations, and by Saito, ${ }^{11}$ including 13230 configurations in a multireference configuration interaction (MRCI). Those calculations recover $93.83 \%$ and $93.84 \%$ of the correlation energy. The CI expansion is slowly convergent, and Bromley and Mitroy found PsH still unbound even including 3457 configurations. The most accurate and reliable variational calculations have been performed using Hylleraas-type functions ${ }^{12}$ and explicitly correlated Gaussians (ECGs). ${ }^{13}$ Quantum Monte Carlo (QMC) results ${ }^{14,15}$ are in agreement with those values. A summary of previous results is reported in Table I.

Functions that do not satisfy the cusp conditions make the convergence very slow, but even the use of explicitly correlated basis sets has resulted in long expansions. Le Sech and $\mathrm{Silvi}^{16}$ tackled the problem of deriving a simple, compact, and accurate wave function for PsH, which could allow a simple physical interpretation of the different terms constituting it, by constraining their wave function to fulfill all the cusp conditions at interparticle coalescence points.

In this paper we show how a wave function constrained to satisfy not only the cusp conditions, but also the correct 
TABLE I. Some of the previous works on PsH.

\begin{tabular}{|c|c|c|}
\hline Type & Energy (hartree) & Reference \\
\hline $\mathrm{SCF}$ & -0.6669 & Strasburger and Chojnacki (1995) ${ }^{\mathrm{a}}$ \\
\hline VMC single term & -0.7723 & Le Sech and Silvi $(1998)^{\mathrm{b}}$ \\
\hline Hylleraas 12 terms & -0.7742 & Lebeda and Schrader $(1969)^{\mathrm{c}}$ \\
\hline VMC single term & -0.7774 & Jiang and Schrader $(1998)^{\mathrm{d}}$ \\
\hline CI 95324 configurations & -0.7867761 & Bromley and Mitroy $(2002)^{\mathrm{e}}$ \\
\hline MRCI 13230 configurations & -0.786782 & Saito $(2003)^{\mathrm{f}}$ \\
\hline Hylleraas 396 terms & -0.788951 & Saito $(2000)^{g}$ \\
\hline ECG 1600 terms & -0.7891965536 & Usukura, Varga, and Suzuki (1998) \\
\hline Hylleraas 5741 terms & -0.7891967 & Yan and Ho $(1999)^{\mathrm{i}}$ \\
\hline DMC & $-0.78918(5)$ & Jiang and Schrader $(1998)^{\mathrm{d}}$ \\
\hline DMC & $-0.78915(4)$ & Mella, Morosi, and Bressanini (1999) \\
\hline VMC single term & $-0.786073(6)$ & Present work \\
\hline${ }^{\mathrm{a}}$ Reference 7. & \multicolumn{2}{|c|}{${ }^{\mathrm{f}}$ Reference 11.} \\
\hline${ }^{\mathrm{b}}$ Reference 16. & \multicolumn{2}{|c|}{${ }^{\mathrm{g}}$ Reference 26.} \\
\hline${ }^{\mathrm{c}}$ Reference 17. & \multicolumn{2}{|c|}{${ }^{\mathrm{h}}$ Reference 13 . } \\
\hline dieference 24. & \multicolumn{2}{|c|}{${ }^{\mathrm{i}}$ Reference 12.} \\
\hline${ }^{\mathrm{e}}$ Reference 10 & \multicolumn{2}{|c|}{${ }^{\mathrm{j}}$ Reference 15 . } \\
\hline
\end{tabular}

asymptotic behavior when a particle goes to infinity, can give better results and allow an easy interpretation of the structure of $\mathrm{PsH}$.

\section{CONSTRUCTION OF THE WAVE FUNCTION}

Within the Born-Oppenheimer approximation, the nonrelativistic Hamiltonian operator for $\mathrm{PsH}$ can be written as

$$
\begin{aligned}
H= & -\frac{1}{2}\left(\nabla_{1}^{2}+\nabla_{2}^{2}+\nabla_{p}^{2}\right)-\frac{1}{r_{1}}-\frac{1}{r_{2}}+\frac{1}{r_{p}}+\frac{1}{r_{12}} \\
& -\frac{1}{r_{1 p}}-\frac{1}{r_{2 p}},
\end{aligned}
$$

where we indicate the electrons with 1 and 2 and the positron with $p$.

Already in their 1969 paper, Lebeda and Schrader ${ }^{17}$ recognized the importance for the wave function to satisfy the Kato cusp conditions. ${ }^{18}$ At particles coalescence, the exact wave function behaves as

$$
\left.\frac{1}{\Psi} \frac{\partial \Psi}{\partial r_{i j}}\right|_{r_{i j}=0}=c,
$$

where $c$ is a constant, depending on the type of the colliding particles. In our case, $c=1 / 2$ for electron-electron, $c=-1 / 2$ for electron-positron, $c=-1$ for electronnucleus, and $c=1$ for positron-nucleus interactions.

The local solution of Eq. (2) suggests that a good trial wave function should have the following asymptotic behavior, when all particles are well separated except one of the leptons is close to the nucleus,

$$
\begin{aligned}
& \Psi(1,2, p) \stackrel{r_{1} \rightarrow 0}{\longrightarrow} e^{-r_{1}} G_{1}\left(r_{2}, r_{p}, r_{2 p}\right), \\
& \Psi(1,2, p) \stackrel{r_{2} \rightarrow 0}{\longrightarrow} e^{-r_{2}} G_{1}\left(r_{1}, r_{p}, r_{1 p}\right), \\
& \Psi(1,2, p) \stackrel{r_{p} \rightarrow 0}{\longrightarrow} e^{r_{p}} G_{2}\left(r_{1}, r_{2}, r_{12}\right),
\end{aligned}
$$

and the following behavior when two leptons are close to each other,

$$
\begin{aligned}
& \Psi(1,2, p) \stackrel{r_{12 \rightarrow 0}}{\longrightarrow} e^{r_{12} / 2} F_{1}\left(r_{1}, r_{p}, r_{1 p}\right), \\
& \Psi(1,2, p) \stackrel{r_{1 p} \rightarrow 0}{\longrightarrow} e^{-r_{1 p} / 2} F_{2}\left(r_{2}, r_{p}, r_{12}\right), \\
& \Psi(1,2, p) \stackrel{r_{2 p} \rightarrow 0}{\longrightarrow} e^{-r_{2 p} / 2} F_{2}\left(r_{1}, r_{p}, r_{12}\right),
\end{aligned}
$$

where $G_{1}, G_{2}, F_{1}$, and $F_{2}$ are unknown functions. Note that since some interparticle distances are zero, it is possible to rewrite the argument of the functions using different variables.

In the same paper, Lebeda and Schrader recognized that the simple orbital description is completely inadequate for $\mathrm{PsH}$, especially for the positronic density, and that the explicit correlation between all particles must be included in the wave function.

The second property that we wish to incorporate into the trial wave function is the correct asymptotic behavior when one of the particles goes to infinity. For large $r_{i}$, to first order, the wave function ${ }^{19}$ behaves as

$$
\Psi \stackrel{r_{i} \rightarrow \infty}{\longrightarrow} \phi(1,2, \ldots, i-1, i+1, \ldots) e^{\beta r_{i}},
$$

where $\beta=-\sqrt{2 E_{i}}, E_{i}$ being the energy required to separate the $i$ th particle, and $\phi$ is the wave function of the residual system.

If the positron goes to infinity, $\mathrm{PsH}$ dissociates into $\mathrm{H}^{-}$ $+\mathrm{e}^{+}$, even if its lowest dissociation channel is $\mathrm{PsH} \rightarrow \mathrm{Ps}$ $+\mathrm{H}$ with an energy threshold of -0.75 hartree. Based on the above conditions, a single-particle function describing the motion of the positron in the field of the $\mathrm{H}$ nucleus should behave as $e^{r_{p}}$ when $r_{p} \rightarrow 0$ [Eq. (3)] and as $e^{\beta r_{p}}$ when $r_{p}$ $\rightarrow \infty$ [Eq. (5)]. For the electrons the situation is different, as an electron going to infinity would leave the $\mathrm{e}^{+} \mathrm{H}$ system which is not bound. ${ }^{20}$ However we can apply Eqs. (3) and (5) to the hydrogen negative ion, so the functions used to build the $\mathrm{H}^{-}$wave function should behave like an exponential $e^{-r}$ 
close to the origin and as $e^{\beta r}$ for $r \rightarrow \infty$, again with two different exponential decays. A functional form $f$ with two different exponential decays, which satisfies both the asymptotic conditions exactly by a particular choice of parameters, is $f(r)=e^{\left(a r+b r^{2}\right) /(1+r)}$. A function with slightly more variational freedom is $f(r)=e^{\left(a r+b r^{2}\right) /(1+c r)}$; in this case the asymptotic condition for $r \rightarrow \infty$ defines only the ratio $b / c$.

As to the pair functions describing the electron-electron and electron-positron pairs, in order to satisfy the cusp conditions in Eq. (4) they should behave, for $r \rightarrow 0$, like exponentials with the appropriate parameter, while for $r \rightarrow \infty$ they must go to a constant value. A Jastrow factor $g(r)$ $=e^{d r /(1+e r)}$ can satisfy these conditions.

Here, we propose a simple wave function that includes all two particle correlations

$$
\begin{aligned}
\Psi(1,2, p)= & \left(1+\hat{P}_{12}\right) f_{1}\left(r_{1}\right) f_{2}\left(r_{2}\right) f_{3}\left(r_{p}\right) \\
& \times g_{1}\left(r_{12}\right) g_{2}\left(r_{1 p}\right) g_{3}\left(r_{2 p}\right),
\end{aligned}
$$

where $\hat{P}_{12}$ is the operator that permutes the two electrons. This functional form has been used with success in the past. $^{21,22}$

In explicit form, the wave function, satisfying all the conditions, reads

$$
\begin{aligned}
\Psi(1,2, p)= & \left(1+\hat{P}_{12}\right) e^{\left(-r_{1}+b_{1} r_{1}^{2}\right) /\left(1+c_{1} r_{1}\right)} \\
& \times e^{\left(-r_{2}+b_{2} r_{2}^{2}\right) /\left(1+c_{2} r_{2}\right)} \\
& \times e^{\left(r_{p}+b_{p} r_{p}^{2}\right) /\left(1+c_{p} r_{p}\right)} e^{\left(r_{12} / 2\right) /\left(1+e_{1} r_{12}\right)} \\
& \times e^{\left(-r_{1 p} / 2\right) /\left(1+e_{2} r_{1 p}\right)} e^{\left(-r_{2 p} / 2\right) /\left(1+e_{3} r_{2 p}\right)} .
\end{aligned}
$$

Let us now examine how this wave function can be simplified constraining it to satisfy the correct asymptotic behavior for dissociation.

\section{A. Asymptotic condition for $r_{p} \rightarrow \infty$}

Let us consider the asymptotic form of our trial function for $r_{p} \rightarrow \infty$. Since $r_{p} \approx r_{1 p} \approx r_{2 p} \rightarrow \infty$, the functions $g_{2}$ and $g_{3}$ become constant:

$$
\begin{aligned}
\Psi(1,2, p) \stackrel{r_{p} \rightarrow \infty}{\longrightarrow} & {\left[\left(1+\hat{P}_{12}\right) e^{\left(-r_{1}+b_{1} r_{1}^{2}\right) /\left(1+c_{1} r_{1}\right)}\right.} \\
& \left.\times e^{\left(-r_{2}+b_{2} r_{2}^{2}\right) /\left(1+c_{2} r_{2}\right)} e^{\left(r_{12} / 2\right) /\left(1+e_{1} r_{12}\right)}\right] e^{b_{p} / c_{p} r_{p} .}
\end{aligned}
$$

The exponent $b_{p} / c_{p}$ of the positronic part in the exact wave function is related to the positron affinity. However, our wave function being an approximated one, we do not expect this relation to hold exactly, and prefer to treat it as a variational parameter and optimize it. The electronic part of the wave function, in square brackets, should describe the hydrogen negative ion. We apply again Eq. (5) to the $\mathrm{H}^{-}$wave function. Letting electron 2 go to infinity and assuming, to fix the ideas, $\left|b_{2} / c_{2}\right|<\left|b_{1} / c_{1}\right|$, we obtain

$$
\Psi(1,2) \stackrel{r_{2} \rightarrow \infty}{\longrightarrow} e^{\left(-r_{1}+b_{1} r_{1}^{2}\right) /\left(1+c_{1} r_{1}\right)} e^{b_{2} / c_{2} r_{2}} .
$$

Since the remaining fragment is a hydrogen atom, we can fix $b_{1}=0$ and $c_{1}=0$. In the exact wave function $b_{2} / c_{2}$ should be directly related to the ionization potential, but again we treat it as a variational parameter. In conclusion the resulting wave function for $\mathrm{H}^{-}$,

$$
\begin{aligned}
\Psi\left(H^{-}\right)= & \left(1+\hat{P}_{12}\right) e^{-r_{1}} e^{\left(-r_{2}+b_{2} r_{2}^{2}\right) /\left(1+c_{2} r_{2}\right)} \\
& \times e^{\left(r_{12} / 2\right) /\left(1+e_{1} r_{12}\right)},
\end{aligned}
$$

has three variational parameters, which we optimize using the variational Monte Carlo method. ${ }^{23}$ The values are $b_{2}=-0.1042, \quad c_{2}=0.4100$, and $e_{1}=0.3257$. The ratio $b_{2} / c_{2}=-0.2541$ must be confronted with the theoretical estimate $\beta=-\sqrt{2 E_{i}}=-0.2353$. Although the wave function is not particularly sophisticated, the corresponding variational energy is -0.52503 (1) hartree, the exact energy being -0.5278 hartree. If we force the wave function to decay with the theoretical value, we lose some variational freedom, and the energy is worse by about 2 mhartree.

\section{B. Asymptotic condition for Ps $\rightarrow \infty$}

When the Ps fragment goes to infinity, it leaves a hydrogen atom, so, in Eq. (7), $b_{1}=0$ and $c_{1}=0$. Furthermore, $r_{2}$ $\approx r_{p} \approx r_{12} \approx r_{1 p} \rightarrow \infty, r_{2 p}=O(1)$, so the asymptotic form of Eq. (7) is

$$
\begin{aligned}
\Psi(1,2, p) \stackrel{P s \rightarrow \infty}{\longrightarrow} & \left(1+\hat{P}_{12}\right) e^{-r_{1}} e^{\left(-r_{2 p} / 2\right) /\left(1+e_{3} r_{2 p}\right)} \\
& \times e^{\left(b_{2} / c_{2}+b_{p} / c_{p}\right) r_{p}} .
\end{aligned}
$$

To recover the correct wave function for this dissociation, we have to impose $e_{3}=0$ :

$$
\begin{aligned}
\Psi(1,2, p) \stackrel{P s \rightarrow \infty}{\longrightarrow} & \left(1+\hat{P}_{12}\right) \Psi(H) \Psi(P s) \\
& \times e^{\left(b_{2} / c_{2}+b_{p} / c_{p}\right) r_{p} .}
\end{aligned}
$$

Once again $\left(b_{2} / c_{2}+b_{p} / c_{p}\right)$, in the exact wave function, is related to the $\mathrm{PsH}$ dissociation energy. We treated these four parameters as variational ones, but it is important that the correct exponential behavior be present in our simple trial wave function. A Gaussian function for example would have a too fast decay.

Having constrained few parameters, the $\mathrm{PsH}$ wave function now is

$$
\begin{aligned}
\Psi(1,2, p)= & \left(1+\hat{P}_{12}\right) e^{-r_{1}} e^{\left(-r_{2}+b_{2} r_{2}^{2}\right) /\left(1+c_{2} r_{2}\right)} \\
& \times e^{\left(r_{p}+b_{p} r_{p}^{2}\right) /\left(1+c_{p} r_{p}\right)} e^{\left(r_{12} / 2\right) /\left(1+e_{1} r_{12}\right)} \\
& \times e^{\left(-r_{1 p} / 2\right) /\left(1+e_{2} r_{1 p}\right)} e^{-r_{2 p} / 2},
\end{aligned}
$$

which can be written as

$$
\begin{aligned}
\Psi(1,2, p)= & \left(1+\hat{P}_{12}\right) \Psi\left(H^{-}\right) e^{\left(r_{p}+b_{p} r_{p}^{2}\right) /\left(1+c_{p} r_{p}\right)} \\
& \times e^{\left(-r_{1 p} / 2\right) /\left(1+e_{2} r_{1 p}\right)} e^{-r_{2 p} / 2},
\end{aligned}
$$

to put in evidence the new variational parameters, which we optimized keeping the $\mathrm{H}^{-}$parameters fixed. The optimized values are $b_{p}=-0.1216, c_{p}=0.3996$, and $e_{2}=0.5225$, and the energy is $-0.782715(8)$ hartree. By simultaneously optimizing also the $\mathrm{H}^{-}$parameters the energy does not decrease. Only including $e_{3}$ does the energy lower to 
$-0.786073(6)$ hartree, the parameter values being $b_{2}=-0.1584, c_{2}=0.5304, b_{p}=-0.1251, c_{p}=0.3810, e_{1}$ $=0.3370, e_{2}=0.3903$, and $e_{3}=0.0277$. In this case the description of the dissociation to $\mathrm{Ps}+\mathrm{H}$ is not correct since $e_{3}$ is not zero; however, its very small value suggests that the dissociation to Ps $+\mathrm{H}$ must be included to correctly build the wave function.

\section{DISCUSSION}

The most accurate wave functions reported in the literature are very long linear expansions, with many variational parameters. It is very difficult to give physical meaning to those functions and extract information on the PsH structure. So it is a challenge to develop a compact, but still accurate wave function. The simple functions proposed up to now to describe the PsH system are not very accurate. Following Lebeda and Schrader, ${ }^{17}$ Le Sech and Silvi ${ }^{16}$ developed a simple wave function treating all the Coulomb interactions and fulfilling all the cusp conditions. The asymptotic behavior of the wave function in the limit of infinite interparticle separations was also taken into account. Their wave function was the product of $\Psi\left(H^{-}\right) \Psi\left(e^{+}\right) \Psi\left(e^{+} e^{-} e^{-}\right)$, but it did not include the asymptotic behavior when one of the particles goes to infinity. It had a total of four variational parameters and after optimization gave an energy of -0.7723 hartree. Jiang and Schrader ${ }^{24}$ chose a wave function similar to ours, but slightly more flexible as to the positron-electron interactions, as Pade' approximants instead of Jastrow factors were used. On the contrary for positron-nucleus interactions they selected a Jastrow function, so their wave function cannot show the correct asymptotic behavior when the positron goes to infinity. They fixed all the cusp conditions, but did not consider the other asymptotic conditions. On the whole they optimized ten parameters using separate variational Monte Carlo (VMC) simulations and recovered only -0.7774 hartree. With a smaller number of parameters, 7 , but a better functional form, we got $-0.786073(6)$ hartree. The importance of choosing a correct functional form is evidenced also by the VMC results we computed in our group ${ }^{21}$ with a basis set of correlated exponentials. Six terms (41 parameters) had to be included to get -0.786310 (11) hartree, a slightly better value than the present $-0.786073(6)$ hartree. Not only does our compact wave function give a worse energy by only 0.7 mhartree than the best wave functions based on the orbital approximation and by 3.1 mhartree than the best result, but its functional form evidences that the PsH structure is mainly made by a $\mathrm{H}^{-}$ion interacting with a positron.

\section{Structure of PsH}

There is no general consensus in the literature whether PsH should be considered similar to a diatomic molecule made by an hydrogen atom with a Ps fragment or similar to an $\mathrm{H}^{-}$ion with a positron added. This is reflected by the fact that sometimes two different notations have been used to indicate the positronium hydride: $\mathrm{H}^{-} \mathrm{e}^{+}$or PsH. Many work- ers have tried to understand how the electrons are distributed in PsH and whether the positron and an electron form a Ps, getting different answers.

Frolov and Smith, ${ }^{25}$ looking at the average values of the interparticle distances, noticed that the electron-electron and positron-nucleus distances are significantly larger than those between each of the positive particles and the nearest electron. This led them to conclude that $\mathrm{PsH}$ is a "cluster that consists of the two neutral systems: the hydrogen atom and the positronium atom." However, they also continued noticing that "the distance between the proton and the second (remote) electron is approximately the same as in the $\mathrm{H}^{-}$ ion, while the distance between the first electron and the positron is approximately the same as the distance between the positron and the remote electron in $\mathrm{Ps}^{-}$." So in terms of three-body clusters $\mathrm{PsH}$ can be represented as a "physical sum" of the $\mathrm{Ps}^{-}$and $\mathrm{H}^{-}$ions. They support this point with the fact that the sum of the energies of $\mathrm{H}^{-}$ $(-0.5277$ hartree $)$ and $\mathrm{Ps}^{-}(-0.2620$ hartree $)$ is, to a very good approximation, the energy of the PsH system.

Usukura, Varga, and Suzuki, ${ }^{13}$ using an explicitly correlated Gaussian expansion that gives practically the exact result, computed not only the average values of the distances among the particles in $\mathrm{PsH}$, but also the corresponding distance distribution functions, to gain more insight into its structure. These results were compared with those in the $\mathrm{H}$ and Ps atoms. They found the average electron-positron distance to be larger than that in the Ps atom, the average electron-nucleus distance to be much larger than in $\mathrm{H}(2.31$ bohrs versus 1.5 bohr), and the electron-nucleus distribution function much broader than in the $\mathrm{H}$ atom. On the whole the interaction between $\mathrm{Ps}$ and $\mathrm{H}$ in $\mathrm{PsH}$ distorts both fragments, so the interpretation of $\mathrm{PsH}$ as $\mathrm{Ps}+\mathrm{H}$ is not supported.

Saito $^{26}$ in a recent paper examined the question looking at various density functions computed by Ho's 396-term Hylleraas-type function. ${ }^{27}$ Examining the electron and positron density functions, and the electron-positron pair density function, he noticed that the electron density in $\mathrm{PsH}$ is similar to the one in $\mathrm{H}^{-}$. However, by looking at the electronic distribution calculated by fixing the coordinate of the positron, he observed that, as the positron is moved away from the nucleus, there is an appearance of the Ps structure. He concluded that "not only $\mathrm{PsH}$ has an atomic structure with a positron added to a hydrogen negative ion, but also a diatomic molecular structure which consists of a hydrogen atom and a positronium," a point of view shared by Bromley and Mitroy ${ }^{10}$ who wrote, "The PsH system consists of a reasonably well-defined Ps atom bound to a $\mathrm{H}$ atom, somewhat similar to a light isotope of the $\mathrm{H}_{2}$ molecule."

Beyond bound-state calculations on $\mathrm{PsH}$, also scattering of Ps by $\mathrm{H}$ can give information on $\mathrm{PsH}$ structure. The importance of the $\mathrm{H}^{-}$channel in obtaining a convergent description of positronium-hydrogen elastic scattering was pointed out by Biswas $^{28}$ and confirmed by Blackwood, McAlinden, and Walters, ${ }^{29,30}$ who found that the inclusion of virtual $\mathrm{H}^{-}$formation has a very substantial influence upon the low-energy scattering.

In our derivation of the $\mathrm{PsH}$ wave function, we explicitly included the correct asymptotic behavior. Our construction 


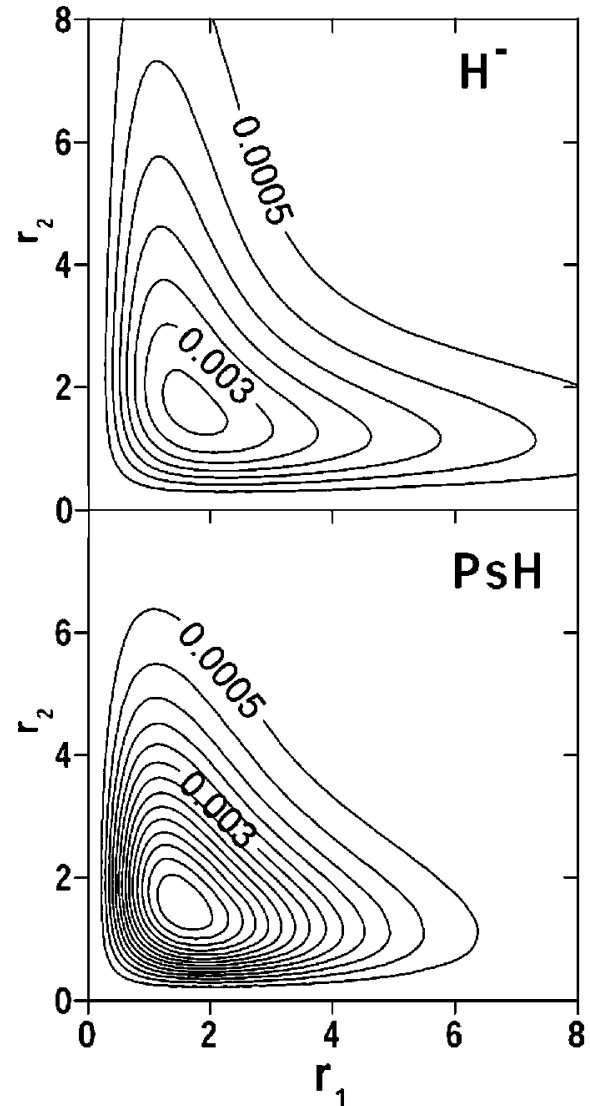

FIG. 1. $\mathrm{H}^{-}$and PsH two-dimensional distribution functions of the electronnucleus distances.

led us naturally to include $\mathrm{H}^{-}$, and this automatically gives a wave function with the character of an $\mathrm{H}^{-}$with a positron bound to it. As correctly observed by Saito, the more distant we put the positron, the clearer is the Ps structure. However, the ground-state wave function is not well approximated by the product of a $\mathrm{H}$ times a Ps. To get a clear view of the electronic distribution, we computed several twodimensional distribution functions for $\mathrm{PsH}$ and $\mathrm{H}^{-}$and we report and discuss the most significant ones. All the distribution functions are normalized to 1 . The two-dimensional electron-electron correlation functions for $\mathrm{PsH}$ and $\mathrm{H}^{-}$are shown in Fig. 1. These correlation functions give more visual information on the electronic distribution around the nucleus than the simple one-dimensional electronic density. From Fig. 1 one can see that the two distributions are very similar, and they both share the characteristic that the highest probability is for both electrons at the same distance from the nucleus, but configurations with an electron close to the nucleus, while the second is further away, are slightly less likely. The addition of a positron to $\mathrm{H}^{-}$keeps the same pattern, shrinking the electron distribution around the nucleus. The distribution of the electron-nucleus distance against the electron-nucleus-electron angle for $\mathrm{H}^{-}$is shown in Fig. 2. When going from $\mathrm{H}^{-}$to $\mathrm{PsH}$ the maximum stays around $100^{\circ}$ for an electron-nucleus distance of about 2 bohrs. To stress the effect of the inclusion of a positron we report the difference between these distribution functions for PsH and $\mathrm{H}^{-}$. The presence of the positron not only shrinks the elec-

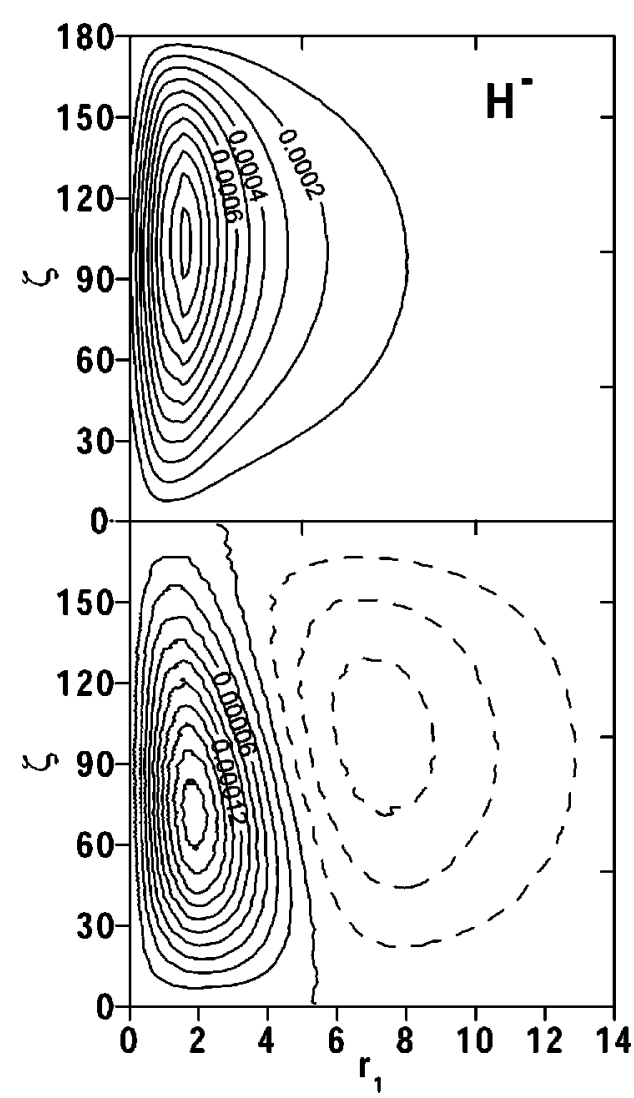

FIG. 2. $\mathrm{H}^{-}$two-dimensional distribution function of the electron-nucleus distance vs the electron-nucleus-electron angle and its change on going from $\mathrm{H}^{-}$to $\mathrm{PsH}$.

tron distribution of $\mathrm{H}^{-}$around the nucleus, as already observed, but the positron, attracting both electrons, also reduces the electron-nucleus-electron angle. To get further inside in PsH structure we computed the distribution of the distances between the positron and the two electrons, shown in Fig. 3. The most likely configuration is for equal distances of the positron from the two electrons, while a Ps $+\mathrm{H}$ structure should give two different electron-positron distances as

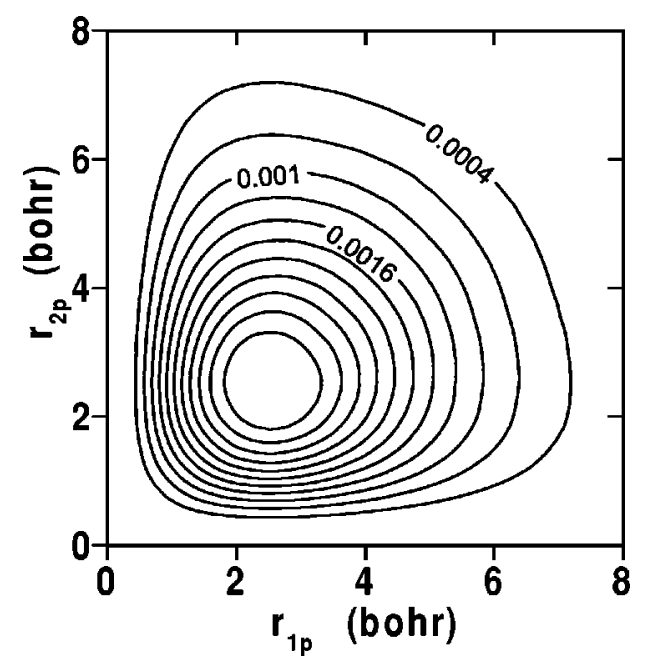

FIG. 3. PsH two-dimensional distribution function of the electron-positron distances. 


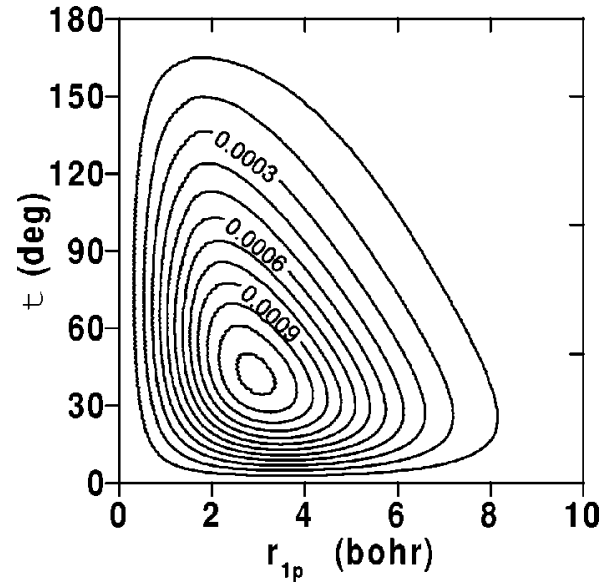

FIG. 4. PsH two-dimensional distribution function of the electron-positron distance vs the electron-positron-electron angle.

the most likely configuration. The distribution of the electron-positron distance versus the electron-positronelectron angle is plotted in Fig. 4: the maximum corresponds to an electron-positron distance of 3 bohrs and an angle of about $40^{\circ}$. As a conclusion PsH cannot be seen neither as $\mathrm{Ps}+\mathrm{H}$ nor as a positron orbiting around $\mathrm{H}^{-}$. Keeping in mind the quantum nature of the leptons and so the impossibility of defining a structure, we suggest to look at PsH as a hydrogen negative ion with the positron that, staying more distant from the nucleus than the electrons, correlates its motion with those of both the electrons. Its attraction on the electrons squeezes them nearer to each other and nearer to the nucleus.

Of course one might object that these distributions might not be representative of the true distributions of $\mathrm{PsH}$, being biased by our choice of the wave function, as our variational Monte Carlo simulations sampled $\Psi_{T}^{2}$. A better, but computationally much more expensive, strategy would be to sample the exact $\Psi_{0}^{2}$ by a forward walking algorithm. ${ }^{23}$ As a computationally cheap compromise we performed standard diffusion Monte Carlo simulations, so sampling $\Psi_{0} \Psi_{T}$, to get a suggestion as to what is missing in our trial wave function. The corresponding distributions show small quantitative, but not qualitative, changes with respect to the previous ones, evidencing the overall correctness of our trial wave function.

\section{CONCLUSIONS}

Choosing a model wave function that can satisfy all the cusp conditions and the asymptotic behavior when a particle goes to infinity we have succeeded in developing a compact, accurate, and physically interpretable wave function for positronium hydride. Optimizing a total of seven parameters we computed an energy $-0.786073(6)$. Our result favorably compares with very long configuration interaction expansions and even with explicitly correlated function expansions. Our wave function and the examination of several two-dimensional distribution functions give new insight into the PsH structure, which is a hydrogen anion perturbed by the positron.

\section{ACKNOWLEDGMENT}

This work has been done in the framework of a project financed by the University of Insubria.

${ }^{1}$ New Directions in Antimatter Chemistry and Physics, edited by C. M. Surko and F. A. Gianturco (Kluver, Dordrecht, 2001).

${ }^{2}$ Positron and Positronium Chemistry, edited by D. M. Schrader and Y. C. Jean (Elsevier, Amsterdam, 1988).

${ }^{3}$ J. Mitroy, M. W. J. Bromley, and G. G. Ryzhikh, J. Phys. B 35, R81 (2002).

${ }^{4}$ D. M. Schrader, in Recent Advances in Quantum Monte Carlo Methods, edited by W. A. Lester, Jr. (World Scientific, Singapore, 1997), Vol. 2, p. 163.

${ }^{5}$ D. Bressanini, M. Mella, and G. Morosi, J. Chem. Phys. 108, 4756 (1998).

${ }^{6}$ M. Mella, M. Casalegno, and G. Morosi, J. Chem. Phys. 117, 1450 (2002).

${ }^{7}$ K. Strasburger and H. Chojnacki, Chem. Phys. Lett. 241, 485 (1995).

${ }^{8}$ A. Ore, Phys. Rev. 83, 665 (1951).

${ }^{9}$ D. M. Schrader, F. M. Jacobsen, N. P. Frandsen, and U. Mikkelsen, Phys. Rev. Lett. 69, 57 (1992).

${ }^{10}$ M. W. J. Bromley and J. Mitroy, Phys. Rev. A 65, 012505 (2002).

${ }^{11}$ S. L. Saito, J. Chem. Phys. 118, 1714 (2003).

${ }^{12}$ Z.-C. Yan and Y. K. Ho, Phys. Rev. A 59, 2697 (1999).

${ }^{13}$ J. Usukura, K. Varga, and Y. Suzuki, Phys. Rev. A 58, 1918 (1998).

${ }^{14}$ T. Yoshida and G. Miyako, Phys. Rev. A 54, 4571 (1996).

${ }^{15}$ M. Mella, G. Morosi, and D. Bressanini, J. Chem. Phys. 111, 108 (1999).

${ }^{16}$ C. Le Sech and B. Silvi, Chem. Phys. 236, 77 (1998).

${ }^{17}$ C. F. Lebeda and D. M. Schrader, Phys. Rev. A 178, 24 (1969).

${ }^{18}$ T. Kato, Commun. Pure Appl. Math. 10, 151 (1957).

${ }^{19}$ U. Kleinekathofer, S. H. Patil, K. T. Tang, and J. P. Toennies, Phys. Rev. A 54, 2840 (1996).

${ }^{20}$ I. Aronson, C. J. Kleinman, and L. Spruch, Phys. Rev. A 4, 841 (1971).

${ }^{21}$ D. Bressanini, M. Mella, and G. Morosi, Phys. Rev. A 57, 1678 (1998).

${ }^{22}$ L. Bertini, D. Bressanini, M. Mella, and G. Morosi, Int. J. Quantum Chem. 74, 23 (1999).

${ }^{23}$ B. L. Hammond, W. A. Lester, Jr., and P. J. Reynolds, Monte Carlo Methods in ab initio Quantum Chemistry, 1st ed. (World Scientific, Singapore, 1994).

${ }^{24}$ N. Jiang and D. M. Schrader, J. Chem. Phys. 109, 9430 (1998).

${ }^{25}$ A. M. Frolov and V. H. Smith, Jr., Phys. Rev. A 55, 2662 (1997).

${ }^{26}$ S. L. Saito, Nucl. Instrum. Methods Phys. Res. B 171, 60 (2000).

${ }^{27}$ Y. K. Ho, Phys. Rev. A 34, 609 (1986).

${ }^{28}$ P. K. Biswas, J. Phys. B 34, 4831 (2001)

${ }^{29}$ J. E. Blackwood, M. T. McAlinden, and H. R. J. Walters, Phys. Rev. A 65, 030502 (2002).

${ }^{30}$ J. E. Blackwood, M. T. McAlinden, and H. R. J. Walters, Phys. Rev. A 65, 032517 (2002). 